\title{
Mobil Cihazlar Üzerinde Enerji Verimli Sanal Sabit Numara Sistemi Araştırma Makalesi/Research Article
}

\author{
Murat BERK ${ }^{1}$, Adnan $\mathrm{OZSOY}^{2}$, (iD İsmail AY ${ }^{1}$ \\ ${ }^{1}$ NETGSM İletişim ve Bilgi. Tek. A.S.., AR-GE Birimi, Ankara, Türkiye \\ ${ }^{2}$ Hacettepe Üniversitesi, Bilgisayar Mühendisliği Bölümü, Ankara, Türkiye \\ murat.berk@netgsm.com.tr, adnan.ozsoy@hacettepe.edu.tr, ismail.ay@netgsm.com.tr \\ (Geliş/Received:16.06.2019; Kabul/Accepted:26.01.2020) \\ DOI: $10.17671 /$ gazibtd. 578489
}

\begin{abstract}
$\ddot{O}_{z}$ et - Kullanıcı alışkanlıklarının mobil cihazlara doğru değişmesi, mobil cihaz yaygınlığının artması ve giderek daha da yaygınlaşması, sabit telefon hizmetlerinin (telefon ve faks) yanı sıra, SMS hizmetinin mobil kullanımı ile farklı bir boyut kazanarak mevcut sabit telefon operatörleri için yeni bir yaklaşım sergilemiştir. Skype ve Whatsapp gibi birçok firmanın benzer özellikte ürün olmasına rağmen, uluslararası geçerli bir numara ile telekom sistemlerine bağlanarak kullanım sunmak internet üzeri yazılı ve sesli iletişim sağlayan önceki saydığımız firmalarda olmayan bir özellik olup, kullanım açısından özellikle kurumsal kullanımlarda fark yaratmaktadır. Kurumsal sabit numara kurumun kimliğinin bir parçasıdır. Bu numara üzerinden çağrı yapmak ve gelen çağrıları almak müşterilerle iletişimde güven sağlar. Bu nedenle özellikle küçük ve orta ölçekli firmalarda, firma çalışanı seyahatte iken kurumsal numara çağrısının o kişiye yönlendirilmesi zor ve ciddi bir maliyet oluşturmaktadır. Bu problemi çözmek için sanal sabit hat uygulamaları geliştirilmektedir. Bu yayın kapsamında mobil cihazlarda sabit telefon özelliklerinin kullanılması, bellek, batarya ve ses kalitesi yönetimi, şifreleme entegrasyonu açısından uluslararası kapsamda özgün çıktıları olan yaklaşımlarla oluşturulmuş mobil cihazlar üzerinde sanal sabit numara sistemi sunulmuştur.
\end{abstract}

\section{Energy Efficient Virtual Fixed Number System on Mobile Devices}

\begin{abstract}
The change in user habits towards mobile devices, the increase in mobile device prevalence, and the widespread use of the mobile devices have gained a new dimension for the existing fixed telephone operators by gaining a different dimension with the mobile use of SMS service as well as fixed telephone services (telephone and fax). Although many companies such as Skype and Whatsapp are products of a similar nature, providing connection to telecom systems with an internationally accepted number is a feature that does not exist in previous companies that provide written and voice communication over the internet. The corporate fixed number is a part of the organization's identity. It provides confidence in communicating with customers to make and receive calls via a fixed number. For this reason, especially in small and medium sized companies, when the employee of the company is traveling, the call for corporate number constitutes a difficult and serious cost to be directed to that person. To solve this problem, virtual landline applications are being developed. Within the scope of this publication, virtual fixed number system has been presented on mobile devices that have been created with approaches that have original outputs in terms of using fixed phone features in mobile devices, memory, battery and voice quality management, encryption integration.
\end{abstract}

Keywords - Telecommunication, Virtual Fixed Number, Energy Efficiency, VOIP 


\section{GİRİS (INTRODUCTION)}

Günümüzde internet ve bilişim teknolojilerinin gelişmesi ile internet protokolleri üzerinden ses (VoIP) teknolojisi yaygınlaşmış olup, sunduğu avantajlar nedeniyle kurumlar veya kişiler, haberleşme maliyetlerini düşürmek amacıyla bu teknolojiden yararlanır. Kurumlara özelleşmiş santraller hem maliyetlerinin uygun olması hem de müşteri ve iç iletişimdeki ihtiyaçların tamamını karşıladığı için daha fazla tercih edilir hale gelmiştir. Özellikle haberleşme açısından yazı ve ses konusunda mobil uygulamalar olarak ortaya çıkmış çözümler, büyük kitlelere ulaşarak çok büyük şirketlerin bünyesine dahil olmuşlardır. Bunlara örnek olarak Skype ve Whatsapp gösterilebilir. $\mathrm{Bu}$ ve benzeri mobil uygulamalar normal telefon kullanımının bile önüne geçebilmekte, her türlü iletişimi bu tür uygulamalar ile gerçekleştirebilmektedirler.

Her ne kadar internet üzerinden iletişim sağlayan mobil uygulamaların kullanımı oldukça sık olsa da, özellikle kurumların sabit hatlı tanımlanan iletişim numaraları da kurumsal kimlik, müşteri güveni, ve yerel bilinirlik açısından önem arz etmekte ve kurumlar tarafindan hala daha günümüzde de sabit hat kullanımı açısından bir talep mevcuttur. Sabit numara üzerinden çağrı yapmak ve gelen çağrıları almak müşterilerle iletişimde güven sağlar. Bu nedenle kullanımı sürdürülmek istenen sabit hatlar için özellikle küçük ve orta ölçekli firmalarda, firma çalışanı seyahatte iken kurumsal numara çağrısının o kişiye yönlendirilmesi zor ve ciddi bir maliyet oluşturmaktadır. Sabit hatları sunulan mekandan farklı olarak hat sağlayıcının kapsamı dışındaki başka bir mekanda sabit hat olarak kullanımı imkan sağlama adına üç temel seçenek vardır. İlki FXS (Foreign Exchange Subscriber - Yabancı Değişim Abonesi) ve FXO (Foreign Exchange Office Yabancı Değişim Ofisi) [1] ile her iki mekanda aracı cihazlar kullanarak internet üzerinden gelen çağrıları karşı tarafta bu cihazlara bağlı telefonlarda bağlayan sistemler mevcuttur. Diğer bir yöntem ise özellikle mobil cihazlar için geçerli, farklı ülkelerdeki servis sağlayıcıların birbirleri ile olan kullanım anlaşmalarına bağlı olarak Roaming teknolojisi ile lokaldeki servis sağlayıcı üzerinden erişim sağlamaktır. Son olarak da VoIP üzerinden numarayı sanallaştırmak ve mobil cihazlarda uygulama ile çağrıların internet üzerinden iletimidir. İlk ikisine nazaran ayrı bir donanım gerektirmemesi ve ücretlendirmelerin çok daha az olması (sadece internet erişimi) nedeniyle üçüncü seçenek çok daha öne çıkmaktadır. Fakat sabit numaraların sanallaştırılarak mobil üzerinde kullanımında bazı sorunlar mevcuttur. Özellikle çağrıları gecikmeden cevaplayabilmesi için programın sürekli arka planda çalışması gerekmekte, sürekli olarak merkeze kayıt olması gerekmektedir. Pil ömrünü maksimize etmek için IOS ve Android işletim sistemlerinin son y1llarda arka planda bildirim sistemleri üzerinden getirdiği kısıtlamalar nedeniyle uygulamanın dinamik değişen internet bağlantıları da düşünüldüğünde sürekli bağlantı ile çağrıları yakalaması önceliğinde detaylı olarak incelenerek yeni sistemlere uyumlu bir sabit numara sanallaştırma sistemine ihtiyaç vardır.
$\mathrm{Bu}$ yayın kapsamında mobil cihazlarda sabit telefon özelliklerinin kullanılması, bellek, batarya ve ses kalitesi yönetimi, şifreleme entegrasyonu açısından uluslararası kapsamda özgün çıktıları olan yaklaşımlarla oluşturulmuş mobil cihazlar üzerinde sanal sabit numara sistemi sunulmuştur. Ayrıca bu yayın kapsamında gerçekleştirilen sanal sabit hat uygulamasi ulusal kapsamda gerçekleştirilen ilk uygulamadır. Yayının geri kalanında ikinci kısımda literatürdeki diğer yayınlar, üçüncü kısımda SIP altyapısı genel bilgi, dördüncü kısımda sunulan sistem modeli, beşinci kısımda sonuç ve ileride yapılabilecek devam niteliğinde çalışmalar ve altıncı kısımda yayın kapsamında alınan destekler sunulmuştur.

\section{LITERATÜR TARAMASI (LITERATURE REVIEW)}

VoIP ve sanal sabit numara üzerine literatürdeki çalışmalar incelendiğinde ilk çalışmalar ile özellikle mobil kullanımın artması ile son zamanlardaki ilgi alanların değiştiğini gözlemledik.

İlk yıllardaki yayınlarda ve patentlerde genel olarak sanal sabit numara sistemine geçiş üzerine yapılan, sistemin alt yapısına yönelik yapılabilirliği üzerine çalışmalar mevcuttur [2-4]. Öncelikli yayınlarda mevcut fiziki numara ve iletişim sistemi üzerinden sanal bir yapıya geçiş için sebepler incelenmiş olup, ekonomik, kullanış kolaylığı, verimlilik ve gelişmekte olan teknolojiler ile uyumu adına incelemeler yapılmıştır [5-9].

Yakın zamanlı çalışmalarda ise yaygınlaşan mobil kullanımı ile mobilin getirdiği farklı problemler ve sorunlar üzerine yoğunlaşıldığı görülmüştür. Özellikle bilgilerin saklanması, iletişimin dinlenebilmesi [10,11], kayıtların ele geçirilmesi [11,13], dolandırıcılık gibi güvenlik açısından mobil cihazlarda has veya daha sıklıkla görülen problemler ortaya çıkmıştır [14-16].

$\mathrm{Bu}$ yayının ana konusu olarak özellikle batarya kullanımı ve ses yönetimi konusunda literatürde VoIP özelinde bazı çalışmalar mevcuttur. [17]'de kablosuz ağ kullanımında uygulamanın uyandırılması üzerine bir çalışma ile enerjide tasarruf sağlamışlardır. [18]'de uygulamanın uyuma moduna alınarak uyuma süresi üzerinde bir optimizasyon ile enerji tasarrufu sağlanmaktadır. Fakat bu iki yayına baktığımızda uyuma temelinde bir geliştirmede uygulamanın bazı çağrıları kaçırma ihtimali vardır. Ayrıca yayınların 2007 ve 2011 tarihli olması nedeniyle mobildeki son gelişmeler ve kısıtlamalara uygunluğu soru işaretidir.

Ses yönetimi adına da literatürde çalışmalar mevcuttur [19-21]. [19]'de akustik ve eko gürültülerinin azaltılması,[20]'de ses ve multimedya aktarımında kaliteyi belirleyici bir standart ve testlerin incelendiği bir teknik rapor, ve [21]'de dinamik olarak kod çözücü seçimi adına dağıtık ve merkeziyetçi iki şekilde bir çalışma sunulmuştur.

$\mathrm{Bu}$ çalışmalara bakıldığında özellikle mobil cihazlardaki batarya kullanımı adına sanal sabit numara alanında 
özelleşmiş ve güncel mobil işletim sistemlerinin kıstaslarıyla entegre bir çalışmaya literatürde rastlanmamıştır. Sıkça kullanılan internet mesajlaşma ve sesli konuşma programlarının (Whatsapp, Skype gibi) en büyük sorunlarından olan bataryanın verimli kullanımı önemli bir çalışma alanıdır. Ayrıca ses kalitesinin kullanıcı merkezinde düşünülerek yönetimi de literatürde açık bir konudur. Dolayısıyla bu yayında özellikle literatürde boşluk olan sanal sabit numara sistemlerinde verimli batarya kullanımı ve ses kalitesinin yönetimi üzerine çalışılmıştır.

\section{SIP ALTYAPISI VE KOD ÇÖZÜCÜLER (SIP INFRASTRUCTURE AND CODECS)}

\subsection{SIP haberleșme altyaptsı (SIP communication infrastructure)}

Mobil platformlarda ses ve iletişim paketlerinin gönderilmesi adına kullanılan en yaygın protokol SIP protokolüdür. SIP (Session Initiation Protocol -Oturum Başlatma Protokolü) VoIP telefon aramalarını oluşturmak, ayarlamak ve bitirmek için kullanılan bir karşılıklı haberleşme protokolüdür. SIP, IETF (İnternet Mühendislik Görev Gücü) tarafindan geliştirilmiş ve RFC 3261 olarak yayınlanmıştır [22]. SIP haberleşmesi oluşturulacak sabit numara sisteminin en temel altyapısını oluşturacağından özellikle açık kaynak kodlu kütüphane araştırılması ve mobil uygulamadaki kullanım şekillerinin araştırılması önemlidir.

\subsubsection{IOS ve Android geliştirme ortamındaki kütüphaneler (Libraries in the iOS and Android development environment)}

SIP haberleşme altyapısında Apple sistemler üzerinde çalışması adına IOS için kütüphanelerden en yaygın olanı PJSIP kütüphanesidir [17]. Ücretsiz ve kaliteli olmas1 nedeniyle çokça tercih edilmektedir. Kütüphanede çok fazla değişken için ayarlar mevcuttur. Apple IPhone telefonlarına uyumlu hale getirme adına birçok ayar kombinasyonu literatürde sunulmuştur. Devam eden araştırmalarımızda Java ve açık kaynak kodlu olan MJSIP ve Sipdroid kütüphaneleri de incelenmiştir [24, 25].

\subsection{Kod çözücü işlemleri ve ses kaliteleri (Codec operations and audio quality)}

Kod çözücüler üzerinde yapılan araştırmalarda genelde C dilinde yazıldığı görülmüştür [26]. Her kod çözücünün kendine göre özellikleri olduğundan, kod çözücülerin farklı olduğu kısımlar hakkında araştırmalar yapılmıştır.

\subsubsection{Kod çözücüler (Codecs)}

Araştırılan kod çözücüler hakkında ulaşılan bilgileri siralamak gerekirse;

G711: U-law ve A-law olarak iki türü vardır. U-law Kuzey Amerika ve Japonya'da daha çok kullanılmaktadır. A-law ise tüm dünyada kullanılıyor. Genelde sistemler her ikisini de desteklemektedir. $64 \mathrm{Kbps}$ hizda seste bozulma yapmadan çalışabilmektedir. Özellikle kablosuz ağda eğer bant genişliği problemi yoksa tercih edilebilir.

G729: S1kıştırma oranı en başarılı olan kod çözücüdür. Ses dosyalarını 8 Kbps'e kadar küçültebilmektedir. Ses kalitesinde ise en az bozulma oranı bu kod çözücüde gözlemlenmiştir. Fakat olumsuz yanlarından biri ücretli bir kod çözücü olmasıdır. Yapılan araştırmada 32 bit sürümlerinin ücretsiz olduğu tespit edilmiştir. ARM temelli işlemcilerde çalışmaktadır.

GSM: Ücretsiz olarak kullanılabilecek en iyi sıkıştırma oranı veren kod çözücüdür. 13 Kbps'e kadar sıkıştırma oranı vermektedir.

G722: HD ses kalitesini 64 Kbps sıkıştırması ile gerekebilmektedir. Android ve IOS tarafinda kararlı ve ücretsiz sürümleri üzerinden ilerlenecektir.

Yapılan kod çözücü araştırmasından sonra bu kod çözücülerin üzerinde çalışacak yazılım ve donanıma özel durumlar ile derlenmesi işlemleri üzerinde çalışıldı. Android de NDK İOS da GCC üzerinde geliştirme yapılabilirliği görüldü. Geliştirme ortamlarında Android için Android Studio, Firebase, Android NDK, Android Support Library, OkHttp, Mjsip, Sipdroid kütüphaneleri, IOS uygulamasi; XCode derleyicisinde, Objective-C dilinde geliştirildi. SIP haberleşmeleri PjSIP kütüphanesi kullanıldı. Uygulama arka planda iken haberleşme için Apple PushKit kütüphanesi kullanıldı. Apple'ın VoIP uygulamalar için özel geliştirdiği ve Apple'ın kendi arama ekranlarının kullanılmasını sağlayan CallKit kütüphanesi kullanıldi.

\subsubsection{Ses titremesi ve gecikme}

Sesteki gecikmelerin ölçülmesi ve ses kalite bozukluğu tespitinden sonra eniyileme işlemleri adına ses paketlerindeki gecikmeleri ölçmek adına sıkça kullanılan ses titremesi (jitter) ve gecikme (latency) değerleri üzerinden hesaplanmaktadır [27]. Eşitlik 1'de bu çalışmada uygulanan hesaplama fonksiyonu verilmiştir. $L$ ( ) etkili gecikme değerini, $x$ gecikmeyi, $y$ ise ses titremesini ifade etmektedir.

$$
L(x, y)=x+2 * y+10.0
$$

Eşitlik 1. Ses titremesi üzerinden gecikme hesaplaması (Calculation of delay over sound shake)

Eşitlik 1'de gösterilen gecikme değeri hesabı üzerinden R, değerlendirme faktörü (rating factor), değeri hesaplanır. $\mathrm{R}$ değeri, gecikme değeri $160.0 \mathrm{~ms}$ altında olanlar için Eşitlik 2, gecikme değeri $160.0 \mathrm{~ms}$ ve üzeri olanlar için Eşitlik 3 üzerinden hesaplanmaktadır.

$$
R=93.2-\left(\frac{L(x, y)}{40.0}\right), \quad L(x, y)<160.0 \mathrm{~ms}
$$

Eşitlik 2. Gecikme değeri $160.0 \mathrm{~ms}$ altında olanlar için R, değerlendirme faktörü, hesaplaması

(Evaluation factor, R, calculation for the delay value below $160.0 \mathrm{~ms}$ ) 
$R=93.2-\left(\frac{L(x, y)-120.0}{10.0}\right), \quad L(x, y) \geq 160.0 \mathrm{~ms}$

Eşitlik 3. Gecikme değeri 160.0 ms ve üstü olanlar için R, değerlendirme faktörü, hesaplaması

(Evaluation factor, R, calculation for delay value $160.0 \mathrm{~ms}$ and above)

Son olarak paket kayıplarını da değerlendirme faktörüne etkisi dahil edilir (Eşitlik 4).

$R=R-2.5 *$ paket_kaybı_yüzdesi

Eşitlik 4. Paket kayıplarını da değerlendirme faktörüne etkisi

(Effect of packet losses on evaluation factor)
Değerlendirme faktörü hesaplanmasından sonra artık MOS, ortalama görüş puanı (mean opinion score), hesaplamasını yapabiliriz. Bu hesaplama için de üç durum söz konusudur. Eğer R değeri 0 dan düşükse, 0 ve 100.0 arasında bir değerse ve son olarak da $R$ değeri 100.0 ve yukarısında bir değerse ayrı hesaplama yapılır. Eşitlik 5 'de bu üç durum için MOS değeri hesabı verilmiştir.

Ses dönüşümü için gerekli seçilen kod çözücü tamponuna göre maksimum ses titremesi (maxjitter) bu fonksiyon üzerinden hesaplanır. Görüşme için SIP sunucu ve kiracı arasında her iki tarafın da uyumlu olduğu kod çözücüler belirlenmektedir. Burada en uygun kod çözücü seçimi kalite ve gecikmeler açısından önemlidir.

$$
\text { MOS }=\left\{\begin{array}{lr}
1.0, & R<0.0 \\
1+0.035 * R+7.10^{-6} * R *(R-60) *(100-R), & 0<R<100 \\
4.5, & R \geq 100.0
\end{array}\right.
$$

Eşitlik 5. MOS ortalama görüş puanı hesaplaması (MOS average opinion score calculation)

Tablo 1. MOS Kalite Tablosu (MOS Quality Table)

\begin{tabular}{|c|c|}
\hline Değer & Etiket \\
\hline \hline 5 & Mükemmel \\
\hline 4 & İyi \\
\hline 3 & Orta \\
\hline 2 & Düşük \\
\hline 1 & Kötü \\
\hline
\end{tabular}

Tablo 1'de MOS değerleri üzerinden kalite tablosu sunulmuştur. Sunulan tabloda hesaplanan MOS değerine bağlı olarak etiketler belirtilmiș olup, pratikte en yüksek MOS değerine G.711 kod çözücü ile 4.5 değer seviyelerinde ulaşılabilmektedir. İletişimde en düşük kabul edilebilir MOS değeri de 2.5 seviyesidir. Bu değerin altındaki bir iletișimin anlaşılabilir seviyenin altında ve kabul edilemez bir seviye olduğu kabul görmüştür [21,27].

\section{SANAL SABITT NUMARA SISTEMI (VIRTUAL FIXED NUMBER SYSTEM)}

Bu kısımda sanal sabit numara sistemi geliștirme adına yapılan çalışmaları ilgili başlıklar altında inceledik. Bu başlıklar sırasıyla mobil üzerinde SIP haberleşmesi, ara yüz, şebekeye entegrasyon, RTP akışı, batarya kullanımı, ses kalitesi üzerine en iyilemeler ve son testlerdir.

\subsection{Mobil platformlarda SIP haberleşmesi (SIP communication on mobile platforms)}

Bölüm 3.1.2'de bahsedildiği üzere haberleşme altyapısında Apple sistemler üzerinde çalışması adına IOS için kütüphanelerden en yaygın olanı PJSIP kütüphanesidir. Ücretsiz ve kaliteli olması nedeniyle çokça tercih edilmektedir. Kütüphanede çok fazla değişken için ayarlar mevcuttur. IPhone telefonlarına uyumlu hale getirme adına birçok ayar kombinasyonu denenip yeniden derlendi. PjSIP kütüphanesinin IOS uygulamalar içerisinde kullanılabilmesi amacıyla derlenmesi sırasında yapılabilen bu ayarları listelemek gerekirse:

- Uygulamanın hedef cihaz işlemcileri mimarisi (armv7, armv7s, armv64, armv64e, i386, x86_64) [28]

- Şifreli sinyalleşme için SSL ve TLS desteği

- Videolu görüşme desteği sunan H264 kod çözücü desteği

- OPUS kod çözücü desteği

- Apple'In 01.06.2016 tarihinden sonra yayınlanan bütün uygulamalar için zorunlu tuttuğu IPv6 desteği [29]

- ZRTP desteği (Zimmermann Real-time Transport Protocol- Canlı telefon görüşmesinin dinlenmesi anlamına gelen "araya giren adam saldırılarına" (Man-inthe-middle-attack-MiTM) karşı güvenlik)

- WebRTC desteği

$\mathrm{Bu}$ listelenen ayarlardan en başarılı bulunan ayarlar ile derlenen kütüphane kullanılmak üzere sisteme kaydedildi. Android üzerinde de PJSIP kütüphanesi denendi ancak bir çok ayar denenmesine rağmen başarılı bir derleme gerçekleştirilemedi. Bu sebeple araştırmalara devam edildi ve tamamen Java ve açık kaynak kodlu olan MJSIP ve Sipdroid kütüphanelerine karar verildi. Özellikle Sipdroid kütüphanesinde bir çok hata tespit edildi ve diğer kullanılan kütüphanelerde uyumsuzlukları tespit edildi. Ancak açık kaynak kod olduğu için tespit edilen yerlerdeki hataların geliştirilerek giderilebileceği düşünüldüğünden kullanılmasına karar verildi.

\subsection{Kullanıc ara yüzleri (User interfaces)}

Kullanıcı ara yüzlerinin oluşturulması kapsamında Android ve IOS üzerindeki geliştirme ortamları incelendi. Dizayn olarak her iki ortam için de genel itibariyle aynı görüntü ve renk düzeni belirlendi. Uygulama ekranları kullanıcı ekranlarına göre gruplara ayrıldı ve tasarlandı. SMS ve arama özellikleri için bir uygulama, faks özellikleri için ayrı bir uygulama olmasına karar verildi. Bu 
ayrımın ana sebebi faks gönderim için kullanılan hattın genelde ayrı olmasındandır.

Uygulama bölümleri olarak;

- Telefon: Arama işlemleri gelen ve giden arama ekranı

- SMS: Gelen SMS, Giden SMS raporları ve yeni SMS gönderme ekranları

- Rehber: Telefon rehberi, Web rehberi, Telefondan web rehbere yükleme, rehberde arama ekranları

- Faks ekranı: Faks gönderme, Faks raporları ve Gelen faks ekranları

- Uygulama ayarları, Hesap ayarları ve diğer ayarlar için ara yüz bulunmaktadır.

Şekil 1'de de görsel olarak hazırlanan taslak ara yüz gösterilmiştir. Android için kullanılacak tüm görsel nesneler çizilebilir obje formatlarında (drawable - hdpi, ldpi, mdpi, xdpi, xxdpi) olarak tasarlanmıştır. Renk düzeni için kullanılacak görseller için tema nesnesi üzerinden bir geliştirme yapılmaktadır. Ekran tasarımları için ana hat düzenleri asgari 5.1 inç ekranlar için tasarlandı. Kullanılacak animasyon nesneleri hazırlandı. Görsel olarak daha kolaylık sağlaması için kütüphane olarak yapılan literatür taraması sonucu Butterknife [30] ve Sweetalert [31] kütüphaneleri öne çıkmaktadır, bu nedenle bu çalışmada da bu kütüphaneler tercih edilmiştir. Aynı zamanda destek kütüphanesindeki "appcompat”, “desin”, "pregerence", "constraint” kütüphaneleri araştırıldı.

Android için kullanılan görseller IOS için de kullanıldı, otomatik ana hat düzenleri (auto layout) halinde IOS'in tüm ekranları için dizayn edildi. Ayrıca görsellik ve kullanım kolaylığı amacı ile MJRefresh, SCLAlertView ve UUChatTableView açık kaynak kodlu kütüphaneleri, veri tabanı olarak da FMDB açık kaynak kodlu kütüphaneleri kullanıld1 [32-36].

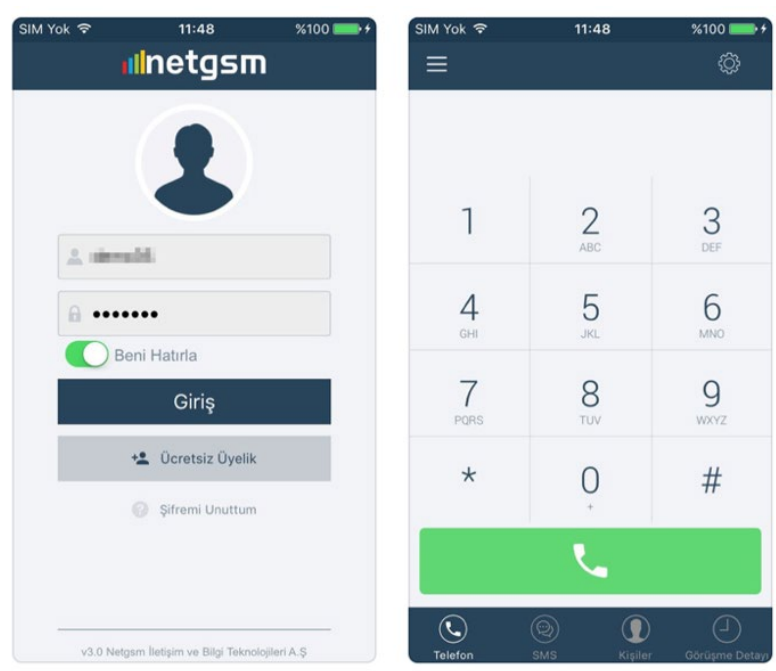

Şekil 1. Uygulama ara yüzü örnekleri (Application user interface samples)
4.3. Telekom şebekesine entegrasyon (Integration to the telecom network)

4.3.1. Sabit Telefon Şebekesine Bağlantı (Connection to Fixed Telephone Network)

Öncelikle abonenin sisteme girişi için bir servis katmanı üzerinden erişim modeli tasarlandı. Abone şifre ile giriş yaptığı zaman sisteme güvenli katmanda (SSL) bir servis üzerinde doğrulama yaparak 6 saatlik bir oturum oluşturulmaktadır. Aynı zamanda abonenin sistem üzerinde yaptığı ses, SMS, faks, ve rehber gibi özelleştirdiği tüm ayarlar sisteme bu süreçte yüklenmektedir. Ses şebekesinin erişim şifreleri farklı olduğu için aboneye tekrar şifre girdirmemek için bu servisten ilgili erişim bilgileri çekilmekte ve kaydedilmektedir.

Ses şebekesine bağlanmak için SIP kütüphanesi üzerinden geçilmesi gerekmektedir ve önceki iş paketinde karar verilen Android için MjSip ve Sipdroid kütüphaneleri sisteme yüklendi. Kütüphanede ile gelen jenerik kullanıcı ara yüzleri yerine geliştirilen yeni ara yüzlere uygunluk sağland.

Kullanılan kütüphanelerin Android yeni sürümlerindeki özellikleri kullanamadığımızı tespit ettiğimizden yeni ara yüzler ile oluşturulan yapı kullanılmıştır. Bu nedenle bir çok altyapı sistemleri ve kullanıcı ara yüzleri yeniden tasarlandı. Özellikle arama yönetim ekranı olan gelen ve giden çağrıları yönetim ekranları üzerinde birçok araştırma yapıldı ve yeniden hazırlanması konusunda bir çok problem çıktı. Örnek olarak Android de kullanıcı telefonu kulağına yaklaştırdığında ekran kilitlenip ekran üzerinde herhangi bir tuşlama yapamaması, yakınlık ve tutuş bilgisine bağlı işlemlerin yapılması gerekli idi.

Kulaktan uzaklaşıldığı zaman tekrar eski haline dönmesi sürecini yönetmek için cihaz üzerindeki yakınlık (proximity) sensörü kullanıldı ancak bunların kullanılabilmesi için Android 7 ve sonrası sürümlerde kullanıcıdan sistem ayarlarına erişim izni alınması gerekti. IOS için de PJSIP kütüphanesi kullanıldı. IOS bu tarz konularda geliştiricileri ve kullanıcıları kısıtladığı için bu tarz işlemler yapılmasına ihtiyaç kalmadı. Kütüphane başarılı bir SIP haberleşmesi sağladı. Gerekli ekranlar ile entegrasyonu yapild1.

\subsubsection{SMS şebekesine bağlanmast (Connecting to the SMS network)}

SMS şebekesine bağlantı için oluşturulan modelde tamamen REST/Json şeklinde bir bağlantı ile erişim tasarlandı (Şekil 2). SMS gönderme, Gelen ve Gönderilen SMS modülleri hazırlandı. SMS gönderimi sırasında ister telefon rehberine istenirse web rehberine SMS gönderim imkanı verildi. Burada yaşanan en büyük zorluk SMS sistemindeki mesaj karakter hesaplama sisteminin aynısının buraya Android ve IOS uyumlu şekilde aktarilması oldu. 
SMS sistemi GSM-7 bit alfabesine göre çalışır ancak akıllı telefonlarda bu kapsamdan daha geniş kullanımda birçok karakter (emoji ve özel karakterler) vardır. Türkçe ve özel karakterleri ekleyebilmek adına Single Shift Character ve Basic Character Set Extension karakter tablosu referans alınarak bir çözüm oluşturuldu ve Türkçe karakterlerin gösterimi ikişer karakter sayıldı.

Aynı zamanda BTK tarafindan mesaj sonuna B021 kodu sistem tarafindan ekleneceği için ülkemizde uygulanan bu yurtdışı standartlarından farklı uygulama abone ara yüzlerindeki karakter hesaplarında özel durumlara neden oldu ve standart dıșına çıkılması ile çözülebildi. Gelen SMS ekranı karşılıklı mesajlaşma şeklinde tasarlandı ve gelen SMS numarası telefon rehberinde kayıtlı değilse web rehberinden sorgulandi.

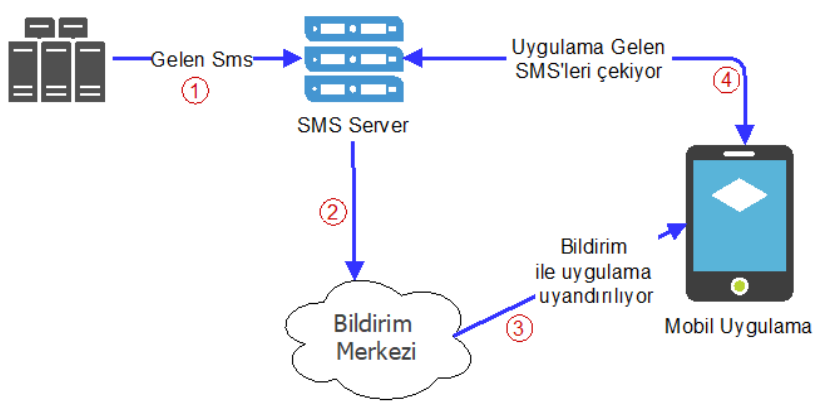

Şekil 2. SMS Şebekesinde Mobil Uygulama (Mobile Application in SMS Network)

\subsubsection{Faks șebekesine bağlantı (Connection to the fax network)}

Faks şebekesine SMS olduğu gibi REST/Json şeklinde bir bağlantı ile bağlanıldı ve her iki sistem içinde merkezi servis yazılımları gerçekleştirildi. Faks göndermek, gelen faks ve gönderilen faks şeklinde 3 bölüm hazırlandı. Faks gönderimi için gönderilecek dosya istenirse telefonda kayıtlı dosyadan (IOS bu özelliği desteklemediği için yalnızca Android üzerinde geçerli) istenirse de kameradan resim çekerek faks gönderimi sağlandı. Burada telefonların kamera kaliteleri ve özellikleri değișken ve yüksek olduğundan gönderilen faks sistemde hata oluşturuyordu bu konuda kaliteyi en az bozan ve çözünürlüğü sistem isterlerine getiren geliștirme yapıldı. Uygulamanın bu bölümü sayesinde IOS ve Android temelli cihazlar aynı zamanda birer faks cihazına dönüştürülmüş oldu.

\subsection{RTP akışl ve Kod çözücü eniyileme (RTP stream and Codec optimization)}

RTP akışları için Android üzerinde AudioManager ve AudioRecord objeleri kullanıldı. Rtpsender ve Rtpreceiver için çalışan sistem üzerinde iş parçacıkları (threadler) oluşturulup, bu iş parçacıklarının telefonun mikrofonundan aldığ sesi iletişim sırasında belirlenen kod çözücü ile kodlama yapılarak oluşan bayt dizini ile RTP paketi oluşturulup gönderilmektedir. Aynı şekilde soketten gelen RTP paketindeki veri bölümü belirlenen kod çözücü ile çözülerek hoparlöre verilmektedir (Şekil 3).

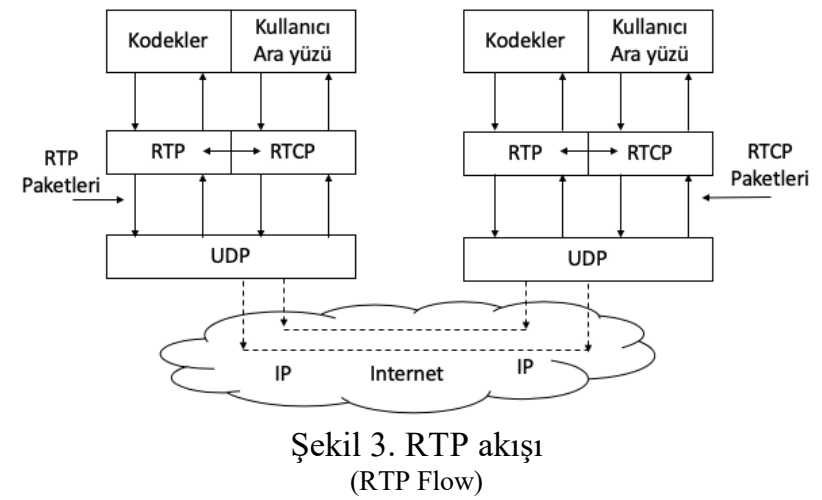

Mikrofondan alınan sesteki yankıları ve arka plan sesi engellemek için NoiseSuppressor AcousticEchoCanceler kütüphaneleri araştırıldı. Yapılan testlerde bu iki kütüphanenin piyasadaki kullanilan tüm telefonlar tarafından desteklenmediği tespit edildi. Yapılan araştırmada Android VOİP işlemleri için audiorecord objesi için "MediaRecorder-AudIOSourceVOICE_COMMUNICATION" modu ile bir çözüm kullanıldı. Bu mod açık olduğu zaman sesteki yankılanma ve arka plan seslerinin engellendiği görüldü.

Gelen RTP ses paketleri ile ilgili jitter (eko titremesi sinyal istikrarsızlığı) ölçümü yapılarak kabul edilebilir eşik olan $30 \mathrm{~ms}$ kriterinin altındaysa ses paketleri işletilmektedir. Daha fazla olan gecikmelerde ses paketleri silinerek/atlanarak istikrarsızlığın diğer paketleri de etkilemesinin önüne geçilerek yeni ses paketleri alınmaya çalışılmaktadır. $\mathrm{Bu}$ sayede sesteki gecikmelerden kaynaklanan konuşan kişinin sesinin gecikerek duyulması önlendi.

4.5. Batarya kullanımında ölçüm ve eniyileme (Measurement and optimization of battery usage)

Mobil platformlarda SIP haberleşmesi için arka planda çalışan sistemlerin harcadığı enerji açısından sorunlar ortaya çıkmaktadır. Uygulamanın arka planda sürekli çalışması yerine daha enerji verimli çözümler üzerinde çalışılmıştır. Öncelikli olarak arka planda sürekli çalışan bir sistemde yapilan testlerde 100-300 mAh kapasiteli batarya kullanımı tespit edilmiştir. Bu batarya kullanımı oldukça yüksektir. Batarya kullanımına neden olan en önemli etken arka planda SIP protokolü için gerekli kullanıcının kayıt ve çevirim içi bilgisinin sürekli gönderilmesidir. Enerji kazanımı adına sürekli gönderim yerine, gönderim sıklığının daha düşük bir seviyede yapılmasına imkan veren bir tasarım düşünülmüştür. Bu ilk tasarımda 60 sn ayarlı olarak testler gerçekleştirildi. Fakat bu çözümde de süre uzadığı zaman oluşan kopmalardan SIP sunucusunun haberdar olmadığı için ilgili cihaza ulaşmaya çalışan sistem belirli bir süre sonra zaman aşımı olarak çağrıyı sonlandırdığı gözlendi. Dolayısıyla, Şekil 4'te gösterildiği gibi bunun da uygun bir çözüm olmadığ1 anlaşıldı. 


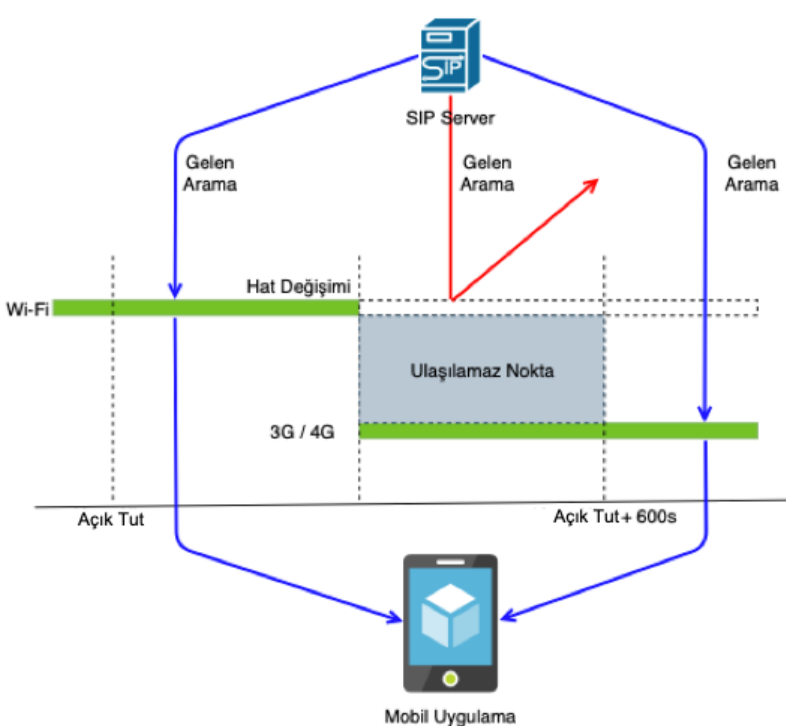

Şekil 4. Arka planda sürekli kayıtlı çalışma durumunu gösterir şekil. Görüldüğü üzere hat değișimlerinde ulaşılamaz bir zaman aralığı mevcuttur. Kayıt ve çevirim içi bilgisinin sürekli gönderilmesi de güç açısından sorun oluşturur. Bu iki yöntem yerine anlık bildirim üzerinden geliştirilen yöntem.

(The figure shows the continuous operating status in the background. As can be seen, there is an unreachable time interval for line changes. Continuous transmission of recording and online information is also a problem in terms of power. Instead of these two methods, the method was developed through push notification.)

İkinci bir tasarım olarak batarya optimizasyonu için yapılan araştırmada Android ve IOS işletim sistemlerinin bu tür durumlar için sunduğu anlık bildirimler (push notification) sistemlerinin kullanılabileceği görüldü. $\mathrm{Bu}$ sistemler IOS ve Android merkezlerine bir bildirim yapıldığı zaman telefondaki uygulamayı uyarabilmesini desteklemektedir. Uygulamanın merkeze sürekli kayıt olmasının nedeni gelen bir çağrı olduğu zaman sunucunun çağrıyı nereye vereceğini bilmesi gerekliliğidir. Anlık bildirim sistemini kullandığımız zaman çağrı geldiğinde uygulamanın merkeze bağlanması sağlanabileceği görüldü. Ancak bunun için çağrı geldiğinde bildirim gönderebilen ve çağrı yapan kişiyi sahte çalma sesi ile beklemeye alan merkezi bir SIP sunucu geliştirildi. $\mathrm{Bu}$ sunucu çağrı geldiği zaman eğer uygulamayı kullanan bir abone ise çağrıyı kendi üzerine alan ve çağrı yapan kişiye sahte çalma sesi veren bir sistem geliştirildi. $\mathrm{Bu}$ arada çağrıyı alacak kişinin IOS veya Android üzerinde olduğu tespit edilip ve ona göre anlık bildirimler gönderilmektedir.

$\mathrm{Bu}$ tasarımda ilgili uygulama telefonu gelen çağrı var şeklinde çalmaya başlıyor ve merkeze kayıt oluyor. Merkez kayıt olan kişiye çağrıyı bağlıyor ve gerçek çalma sesine dönüştürüyor. Bu sayede merkeze sürekli kayıt gönderme ihtiyacı kalmıyor (Şekil 5). Ancak bu senaryoda da olası internet durum değişikliklerinde veya uygulamanın tamamen kapanması durumları sorun teşkil etmektedir. Bu tür durumlar için de bir servis geliştirildi. Bu servis çalışmadığ 1 zaman uygulamalar anlık bildirimler alamadığı görüldü.

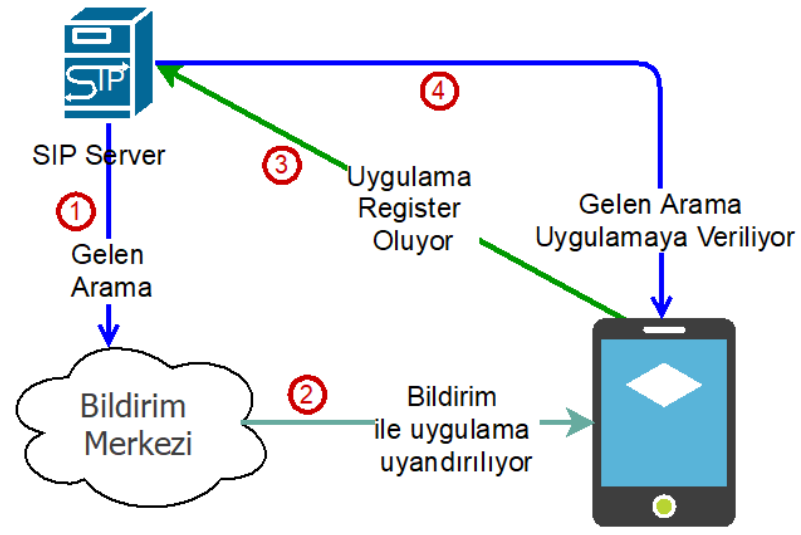

Mobil Uygulama

Şekil 5. Anlık bildirim Metodu ile Çalışma Durumu (Operating Status with Push Notification Method)

Serviste internet bağlantılarında değişiklikler ve telefondaki çağrı durumundaki değişiklikler takip edilmeye başlandı. Örneğin uygulamanın kurulu olduğu telefon kendi şebekesinde bir çağrı yapıyorsa meşgul olduğundan gelen çağrı almaması gerekiyor veya internet bağlantı durumu daha düşük seviyeye (edge) dönüştüyse kaliteli bir ses gönderimi sağlanamayacağından gelen çağrının reddi gibi olasılıklar çalışıldı. Kullanıcının isteğine bağlı olarak sürekli kayıt modu da eklendi. Sürekli kayıt modundan çağrı alınabilmesi ve batarya kullanımını azaltmak için kayıt süresini 600 saniyeye çıkarıldı. Ancak olası internet problemlerinde örneğin kablosuz ağda iken veri planına yani $3 \mathrm{G}$ veya $4 \mathrm{G}$ 'ye geçilmesinde merkeze ayrıca kayıt göndermek suretiyle güncel bağlantı noktasını sürekli bildirilmesi sağlandı.

Tablo 2. Kayıt modu ve batarya kullanımı oranları (Recording mode and battery usage rates)

\begin{tabular}{|l|l|}
\hline \hline Süre(sn) & Batarya Kullanımı (mAh) \\
\hline \hline 60 & $200-300$ \\
\hline 300 & $100-250$ \\
\hline 600 & $50-80$ \\
\hline Anlık bildirim & $1-2$ \\
\hline
\end{tabular}

4.6. Ses Kalite ölçüm ve eniyileme (Sound Quality measurement and optimization)

Yapılan testlerde anlık bildirimler ile çağrı olduğunda bağlan modunda iken batarya kullanımı 1-2 mAh arasına düştüğü görüldü. Süreli kayıt modunda ise 50-80 mAh lık bir kullanım tespit edildi. Dolayısıyla ilk aşamada ölçülen değerler olan 100-250 mAh değerlerinin çok altına düşülmüş ve bu sayede mobil cihaz üzerinde kullanıcı tarafında oluşabilecek pil ömrüne bağlı hoşnutsuzlukların önüne geçilmiş oldu (Tablo 2).

Sesteki gecikmelerin ölçülmesi ve ses kalite bozukluğu tespitinden sonra eniyileme işlemleri adına ses paketlerindeki gecikmeleri ölçmek adına sıkça kullanılan ses titremesi (jitter) ve gecikme (latency) değerleri üzerinden bir yöntem kullanıldı. Ses dönüşümü için gerekli seçilen kod çözücü tamponuna göre maksimum ses titremesi (maxjitter) hesaplandı. Aynı zamanda gönderilen paketin elimize ulaşma zamanındaki gecikme veya 
paketler arası ortalama gecikme süreleri hesaplanarak ses kalitesi etkilenmemesi için çalışmalar yapıldı. Görüşme başında protokol gereği SIP sunucu ve kiracı arasında her iki tarafin da uyumlu olduğu kod çözücüler belirlenmektedir. Burada en uygun kod çözücü sıralamada en üstte olana göre belirlenir.

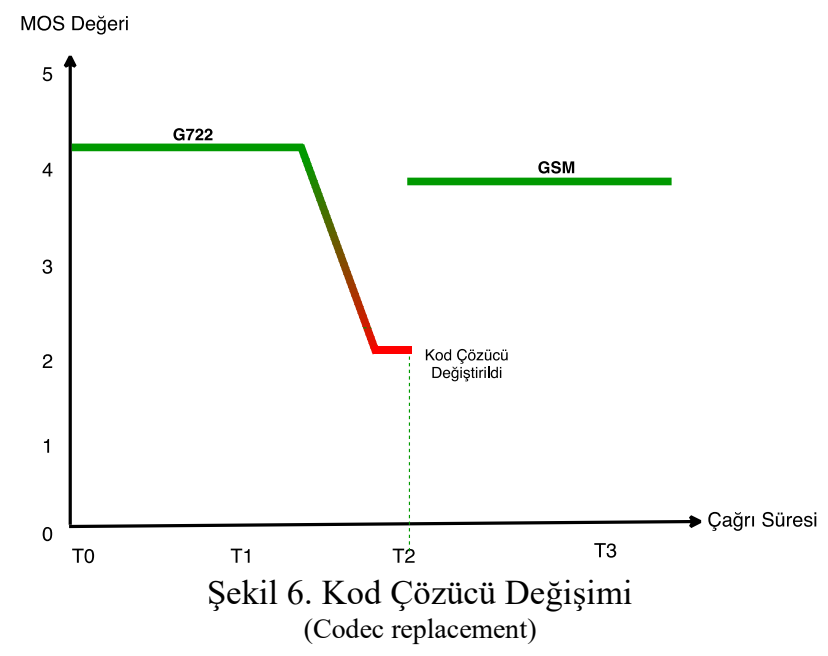

Geliştirilen sistemde kullanıcının bulunduğu ağdaki çekim ve ses kalitesine göre kod çözücü seçimi yapılmaktadır. Örneğin kablosuz çekim kalitesi iyi ve üzeri bir ağda iken yüksek kalite HD kod çözücü seçimi yapılması, kablosuz çekim kalitesi düşük veya $3 \mathrm{G}-4 \mathrm{G}$ bir ağda ise sıkıştırma oranı daha yüksek bir kod çözücü seçimi yapılmasıdır (Şekil 6). Görüşme sırasında eğer görüşme kalitesinde bozulma varsa eldeki kod çözücü kalite sıralamasındaki bir alttaki kod çözücü kullanılması ve bunun SIP sunucuya bildirilmesi üzerine çalışmalar yapıldı. Kod çözücü sıralamalarında sıkıştırma ve kalite oranına göre kod çözücü sıralamaları Tablo 3'de verilmiştir.

Tablo 3. Kod çözücü sıralamalarında sıkıştırma ve kalite oranına göre kod çözücü sıralamaları

(Codec rankings according to compression and quality ratio in decoder rankings)

\begin{tabular}{|c|c||c||c|}
\hline Kod Çözücü & $\begin{array}{c}\text { Paket } \\
\text { Boyutu }\end{array}$ & $\begin{array}{c}\text { Örneklem } \\
\text { S1klığ } 1\end{array}$ & Bant Türü \\
\hline \hline G722 HD & $64 \mathrm{~KB}$ & 16000 & Geniş bant \\
\hline Alaw-Ulaw & $64 \mathrm{~KB}$ & 8000 & Dar bant \\
\hline GSM & $13 \mathrm{~KB}$ & 8000 & Dar bant \\
\hline G729 & $8 \mathrm{~KB}$ & 8000 & Dar bant \\
\hline
\end{tabular}

RTP paketlerinin teslim durumlarının tespiti ve kalite oranının karşı sunucuya bildirilmesi için RTCP protokolü kullanılabilir. Teslim edilen paketlerdeki gecikme sürelerinin öğrenilmesi işlemi, RTCP ile karşı sunucuya sorulabilmektedir. Yukarıda verilen ölçümler tamamen teslim aldığımız paketler üzerinden yapılmaktadır. Fakat, RTCP sayesinde ise teslim ettiğimiz paketlerdeki ses titremesi ve gecikme değerlerini de öğrenebiliyoruz. Görüşme sırasında kod çözücü değişikliği için teslim aldığımız paketler kadar gönderdiğimiz paketlerin ulaşım oranları da önem arz etmekte. Bu bilgiyi kullanarak karşı tarafta oluşan gecikmelere göre anlık bir iyileştirme sunma ve kod çözücü değişimi kararı verilebilir. Sesteki gecikme 10 saniyede ortalama $100 \mathrm{~ms}$ üzerine ise kod çözücü değiştirme kararı veriliyor. Bir insanın $70 \mathrm{~ms}$ üzerindeki gecikmeleri algılayabildiği bilinen bir standarttır. Dolayısıyla, bunu gözeterek $100 \mathrm{~ms}$ üzerinde sesteki bozulma artık iletişimi engellemeye başlamaktadır. Kurduğumuz ve yaptığımız araştırma ile bu paket gecikmelerinde adaptif bir şekilde farklı kod çözücüler ve ağ bilgisi üzerinden eniyileme işlemleri gerçekleştirmekteyiz.

\subsection{Test ve Optimizasyon (Test and Optimization)}

Yapılan çalışmalarda ortaya çıkan durumların genel testleri gerçekleştirilmiştir. Bu test kalemleri ve test sonuçlarını siralamak gerekirse:

- Uygulamaya Giriş: Kullanıcı adı ve şifre doğrulamasının başarılı, başarısız veya bilgilerin boş olduğu durumlar test edildi. Yapilan testler sonucunda hataya rastlanmamıştır.

- SMS Gönderim Testi: Telefon veya web rehberinde yer alan numaralara, web rehberinde yer alan gruplara ya da elle yazılabilecek numaralara, aboneliğe ait abone numarası veya sistemde tanımlı gönderici adı kullanılarak, hemen ya da ileri bir tarihte gönderilmek üzere mesaj oluşturulması işlemidir. Yapılan testlerde herhangi bir hataya rastlanmamıştır.

- SMS Alma testi: Diğer operatörlerden aboneliğe tanımlı numaraya gönderilmiş olan mesajların sunucudan çekilerek mobil uygulama üzerinden gösterildiği ekrandır. Eğer mesajı gönderen mesaj gönderilebilecek bir numara ise gelen mesaj aynı ekrandan cevaplanabilir. Yapılan testler sonucunda hataya rastlanmamıştır.

- Arama Testi: Aramanın başlaması, cevaplanmaması, reddedilmesi, arama sırasında yakınlık sensörü vasıtasıyla ekranın kapatılması, ses kalitesinin düşmesi sonucunda kod çözücü dönüşümü yaparak kalite artırma ve aramanın sonlanması sonrasında arama kayıtlarının oluşturulması testleri hem gelen hem de giden yönlü aramalar için yapıldı. Bunlara ek olarak arama bekletme, mikrofonu sessize alma, DTMF gönderme, bluetooth aygıtlar ile bağlantı gibi testler de yapıldı. Yapılan testler sonucunda hataya rastlanmamıştır.

- Farklı IOS ve Android sürümlerinde; Android'de 4.4 ve IOS'da 7.1 sonrasında çıkan tüm sürümlerde test yapilmıştır.

Yapılan bir diğer test ise farklı mobil kullanım durumları ve bu durumlarda enerji ve kaynak tüketimi üzerinedir. Bu testlerde iki farklı telefon kullanılmıștır: Huawei P10 (Android Pie 8.1), Phone - 2 : iPhone 8Plus (iOS 13.1).

Testler; Wi-Fi, $3 \mathrm{G} / 4 \mathrm{G}$ ve $2 \mathrm{G}$ şebekelerinde, her iki telefonda da yapılmış ve elde edilen ortalama değerler tablolarda gösterilmiştir (Tablo 4 ve 5) . Wi-Fi testlerinde sinyal seviyesi yaklaşık olarak 'Mükemmel' $(-30 \mathrm{dBm})$ ve 'Normal' (-70dBm) seviyelerinde yapılmıştır. 
Tablo 4. Huawei cihazı üzerinde farklı mobil kullanım durumları için MOS ve internet kullanımı testleri (MOS and internet usage for different mobile use cases on Huawei device)

\begin{tabular}{|l|c|c|c|c|c|c|}
\hline \multicolumn{7}{|c|}{ Huawei P10 (Android Pie 8.1) } \\
\hline \multirow{2}{*}{ Kodek } & \multicolumn{2}{|c|}{ Wi-Fi } & \multicolumn{2}{c|}{ 3G/4G } & \multicolumn{2}{c|}{$2 \mathrm{G}$} \\
\cline { 2 - 7 } & MOS & MB & MOS & MB & MOS & MB \\
\hline PCMU & 4,38 & 0,33 & 4,37 & 0,30 & 2,37 & 0,29 \\
\hline PCMA & 4,39 & 0,32 & 4,37 & 0,29 & 2,09 & 0,33 \\
\hline G722 & 4,39 & 0,23 & 4,37 & 0,30 & 2,50 & 0,29 \\
\hline GSM & 3,27 & 0,06 & 3,22 & 0,06 & 1,71 & 0,06 \\
\hline G729 & 3,81 & 0,25 & 3,51 & 0,22 & 1,09 & 0,05 \\
\hline
\end{tabular}

Yapılan testler sonucunda arka planda iken gelen çağrıyı, gelen mesajları ve gelen faksları almak için uyanık kalmak için harcanan batarya miktarı, yükleme (upload) ve indirme (download) miktarları tabloda gösterilmiştir (Tablo-6). Yapılan testlerde 24 saatlik süre diliminde uygulama bekleme modundadır. Başka herhangi bir tüketim yapmamıştır. Anlık bildirim sisteminde çağnı anında uygulama uyandırıldığı için batarya ve internet kullanımı minimum seviyeye indirilmiştir.

Tablo 5. iPhone cihazı üzerinde farklı mobil kullanım durumları için MOS ve internet kullanımı testleri

(MOS and internet usage for different mobile use cases on iPhone device)

\begin{tabular}{|l|r|r|r|r|r|r|}
\hline \multicolumn{7}{|c|}{ iPhone 8Plus (iOS 13.1) } \\
\hline \multirow{2}{*}{ Kodek } & \multicolumn{2}{|c|}{ Wi-Fi } & \multicolumn{2}{|c|}{$3 \mathrm{G} / 4 \mathrm{G}$} & \multicolumn{2}{c|}{$2 \mathrm{G}$} \\
\cline { 2 - 8 } & MOS & MB & MOS & MB & MOS & MB \\
\hline PCMU & 4,38 & 0,31 & 4,35 & 0,29 & 2,12 & 0,30 \\
\hline PCMA & 4,38 & 0,30 & 4,35 & 0,29 & 1,98 & 0,30 \\
\hline G722 & 4,22 & 0,25 & 4,35 & 0,31 & 2,26 & 0,32 \\
\hline GSM & 3,02 & 0,05 & 3,13 & 0,07 & 1,55 & 0,05 \\
\hline G729 & 3,80 & 0,26 & 3,50 & 0,24 & 1,59 & 0,05 \\
\hline
\end{tabular}

Tablo 6. Farklı senaryolar için harcanan batarya miktarı, yükleme ve indirme miktarları (For different scenarios the amount of battery spent, the upload and the download amount)

\begin{tabular}{|c|c|c|c|c|}
\hline & Kullanılan Batarya (mAh) & Yükleme (Kb) & İndirme (Kb) & Zaman Periyodu (sn) \\
\hline \multirow{3}{*}{$\begin{array}{c}\text { Gelen çağrıyı almak için } \\
\text { uyanık kalma }\end{array}$} & $260-270$ & $2370-2400$ & $1565-1750$ & 60 \\
\cline { 2 - 5 } & $50-60$ & $470-490$ & $305-325$ & 300 \\
\cline { 2 - 5 } & $25-30$ & $230-240$ & $150-175$ & 600 \\
\hline \multirow{3}{*}{ Gelen SMS Kontrolü } & $280-300$ & $3425-3500$ & $2980-3010$ & 60 \\
\cline { 2 - 5 } & $50-60$ & $680-700$ & $590-610$ & 300 \\
\hline \multirow{3}{*}{ Gelen Faks Kontrolü } & $25-30$ & $340-350$ & $280-300$ & 600 \\
\cline { 2 - 5 } & $270-290$ & $3250-3300$ & $2770-2800$ & 60 \\
\hline Anlık Bildirim Sistemi & $45-55$ & $650-680$ & $540-560$ & 300 \\
\hline
\end{tabular}

\section{SONUÇ (CONCLUSION)}

Yayın kapsamında sabit hatlı tanımlanan iletişim numaralarının VoIP teknolojisi kullanılarak mobil cihazlarda sabit telefon özelliklerinin kullanılmasına yönelik bir çalışma sunulmuştur. Bu özellikleri sağlama adına bellek, batarya ve ses kalitesi yönetimi, şifreleme entegrasyonu başlıkları incelenmiş olup, uluslararası kapsamda özgün çıktıları olan yaklaşımlarla oluşturulmuş mobil cihazlar üzerinde sanal sabit numara sistemi sunulmuştur.

Özellikle akıllı cihazların her geçen gün artan enerji verimli olma kısıtlamalarına en güncel kıstaslarda özgün bir çözüm geliştirilmiş olup bunun üzerine SIP haberleşme altyapısı, dinamik kod çözücü işlemleri, ses kalite eniyileme, kullanıcı ara yüzleri, ve Telekom şebekesine entegrasyon alanlarında geliştirmeler yapılmıştır. $\mathrm{Bu}$ çalışma ile mobil cihazlarda sabit numara kullanımı sanallaştırılarak, sabit bir mekana zorunlu kalmayıp tüm ticari ve özel işlemlerin internet erişimi olan her yerde kullanımını sağlamaktadır.

\section{DESTEK (ACKNOWLEDGEMENT)}

Bu proje Sanayi Bakanlığı'nın Ar-Ge merkezi bünyesinde sağlanan desteklerle ve TÜBITAK TEYDEB 7180242 numaralı 1507 Kobi-ArGe projesi kapsamında gerçekleştirilmiştir.

\section{KAYNAKLAR (REFERENCES)}

[1] Internet: https://www.3cx.com/pbx/fxs-fxo/, 10.03.2019.

[2] T. H. Ahn, K. Hoon-Bae, K. C. Park, "Method and device for providing mobile services with virtual number", U.S. Patent Application 11/205,976, 2005.

[3] L. Holder, "Method and apparatus for placing a long distance call based on a virtual phone number", U.S. Patent No. 7,386,111. 2008.

[4] J. D. Logan, "Call forwarding system and method employing virtual phone numbers associated with landline and other discrete telephone units", U.S. Patent Application No. 12/344,073, 2010. 
[5] B. Goode, "Voice over internet protocol (VoIP)", Proceedings of the IEEE, 90(9), 1495-1517, 2002.

[6] T. T. Kwon, M. Gerla, S. Das, "Mobility management for VoIP service: Mobile IP vs. SIP", IEEE Wireless communications, 9(5), 66-75, 2002.

[7] B. Massimo, F. Cacace, G. Iannello, M. Vellucci. "Mobility management for VoIP on heterogeneous networks: evaluation of adaptive schemes", IEEE Trans. on Mobile Computing, 6(9), 1035 1047, 2007.

[8] C. Guo, et. al., "End-system-based mobility support in IPv6", IEEE Journal on Selected Areas in Communications, 23(11), 2104-2117, 2005.

[9] C. Steeh, L. Piekarski, "Accommodating new technologies: Mobile and VoIP communication", Advances in telephone survey methodology, 423-446, 2007

[10] Y. Cha, W. Pak, "Protecting contacts against privacy leaks in smartphones", PloS one, 13(7), 2018

[11] C. Çakır, H. Kaptan, "VoIP Teknolojilerinde Opnet Tabanlı Güvenlik Uygulaması", Bilişsim Teknolojileri Dergisi, 2(3), 1-7, 2009.

[12] S. Toklu, O. Erdem, "Idle ve Power_Down Güç Durumunun Enerji Başarım Değerlendirmesi", Bilişim Teknolojileri Dergisi, 5(3), 3540, 2013.

[13] A. Zingerle, "Remain Anonymous, Create Characters and Backup Stories: Online Tools Used in Internet Crime Narratives", Int. Conference on Interactive Digital Storytelling, Springer, 2014.

[14] D. R. Kuhn, T. J. Walsh, S. Fries. "Security considerations for voice over IP systems", NIST special publication, 800, 2005.

[15] A.D. Keromytis, "A comprehensive survey of voice over IP security research", IEEE Comm. Surveys \& Tutorials, 14(2), 514 537,2011

[16] M. Yüksel, N. Öztürk. "SIP Saldırıları ve Güvenlik Yöntemleri", Bilişim Teknolojileri Dergisi, 10(3), 301-310, 2017.

[17] Y. Agarwal, R. Chandra, A. Wolman, P. Bahl, K. Chin, R. Gupta, "Wireless wakeups revisited: energy management for VOIP over wi-fi smartphones", Proceedings of the5th international conference on Mobile systems, applications and services, ACM, 2007.
[18] S. B. Jang, Y. G. Kim. "An energy-efficient delay reduction technique for supporting WLAN-based VoIP in SmartPhone", Journal of Systems Architecture, 57(10), 934-944, 2011

[19] M. Fukui, "Acoustic echo canceller software for VoIP hands-free application on smartphone and tablet devices", IEEE Transactions on Consumer Electronics, 60(3), 461-467, 2014

[20] ETSI, EG. 202 396-1,"Speech and multimedia transmission quality (STQ)" 2011.

[21] A. Sfairopoulou, C. Macián, B. Bellalta, "Dynamic measurementbased codec selection for VoIP in multirate IEEE 802.11 WLANs", Proceedings of the 8th COST 290 Management Committee Meeting, 2007.

[22] J. Rosenberg, "IETF RFC 3261-SIP: Session Initiation Protocol", The Internet Society, 2002.

[23] Internet: https://www.pjsip.org/, 10.03.2019.

[24] Internet: http://sipdroid.org/, 10.03.2019.

[25] Internet: http://www.mjsip.org/, 10.03.2019

[26] Internet: https://www.gl.com/voice-codecs.html, 10.03.2019.

[27] Internet: https://netbeez.net/blog/impact-of-packet-loss-jitter-andlatency-onvoip/, 10.03.2019.

[28] Internet: https://en.0wikipedia.org/wiki/Appledesigned_processors, 10.03.2019.

[29] Internet: https://developer.apple.com/support/ipv6, 10.03.2019.

[30] Internet: http://jakewharton.github.io/butterknife/, 10.03.2019.

[31] Internet: https://sweetalert.js.org/, 10.03.2019.

[32] Internet: https://github.com/rafaelmotta/react-native-scl-alert, 10.03.2019

[33] Internet: https://github.com/ccgus/fmdb, 10.03.2019.

[34] Internet: https://github.com/dogo/SCLAlertView, 10.03.2019.

[35] Internet: https://github.com/CoderMJLee/MJRefresh, 10.03.2019.

[36] Internet: https://github.com/ZhipingYang/UUChatTableView, 10.03.2019. 